\title{
Surgical/Theatre
}

\section{Safety in surgery: human factors}

\section{Sam McMillan}

What are human factors in healthcare? 'Enhancing clinical performance through an understanding of the effects of teamwork, tasks, equipment, workspace, culture and organisation of human behaviour and abilities and application of that knowledge in clinical settings (Catchpole, 2010).

It is an unfortunate truth that most of the significant complications that are encountered during or following a surgery are predictable, and worse still that a good proportion of them are preventable. Essentially, far from being inevitable problems caused by patient morbidity, drugs or equipment, they are problems caused by the surgeon or theatre team, and more specifically by human error. Mistakes and errors are part of human nature, novice and expert alike; they are to be expected and steps should be put in place to predict them. Humanfactors approaches underpin the evidence-based approach required to evaluate patient safety and quality improvement. The more that human performance can be optimized through recognizing human limitations, the more errors can be minimized.

Errors can be made in every part of the surgical process, from the patient assessment and surgical planning, through to the anaesthetic and surgery themselves and into the recovery period. Common errors in surgery include: forgetting to check the patient, incorrect patient, incorrect surgical site identified/clipped/operated on, forgetting a vital piece of equipment or missing a step in a process, and leaving swabs or instruments inside a body cavity. A number of factors can lead reduced levels of cognitive and physical performance and subsequently induce errors: lack or knowledge, skills or experience, distraction stress, fatigue, illness, overwork, time constraints, problem patient/client, understaffing and communication problems are a few of the more commonly encountered factors. These are all commonly encountered in veterinary practice. Recognizing these human factors as significant barriers to effective performance is the first step in trying to reduce errors. To create safe and reliable systems in veterinary practice, we have to base these on an understanding that we must anticipate when things may go wrong and build in suitable defences.

Mechanisms that have been proven to reduce the risk of surgical error based on human factors include: marking the surgical site, recognizing the environmental factors and the importance of using World Health Organization-based surgical checklists.

This lecture will focus on human factors in the surgical environment, the human risk factors for surgical nursing and mechanisms to reduce these.

\section{KEY LEARNING OBJECTIVES}

- Recognize that human error contributes to a huge proportion of error in surgery

- Recognize human factors as significant barriers to effective performance

- Evaluate what can be done to mitigate human factors in surgical error

\section{MULTIPLE CHOICE QUESTIONS}

1. Which of the following statements regarding latent errors is FALSE?
(A) Latent errors are due to individual negligence
(B) Latent errors are 'intrinsic flaws'
(C) Latent errors are 'holes' in safety mechanisms
(D) Latent errors are 'accidents waiting to happen'

2. Which of the following is FALSE? Checklists in surgery have been shown to:
(A) Considerably increase procedure time
(B) Reduce infections associated with central-line placement
(C) Reduce surgical fatality rates
(D) Reduce surgical complications

3. Which of the following statements regarding surgical errors is FALSE?
(A) Indicates a lack of care and attention on behalf of the veterinary nurse
(B) Are often caused by cognitive biases
(C) Are often associated with systems factors
(D) Are commonly encountered

\section{Look after your kit and it will look after you}

\section{Alison Young}

Veterinary practices invest greatly in instruments and equipment and require them to be available and fully functioning at all times. Unlike our human colleagues, we often do not have access to multiples of the same item, so if it is out of action that has a detriemental effect on our patients. The cost of replacement is far greater than the cost of maintaining them, so this must be factored in as part of the normal working day. As part of the nurse's role it is important that instruments are used appropriately - the right instrument for the right job!

\section{CLEANING OF INSTRUMENTS}

Proper care of surgical instruments begins with proper cleaning. This is often seen as a dull time-consuming task and is often pushed to the bottom of the 'to-do' list when there are patients that need nursing care and procedures. However, as previosuly mentioned, this is a key task 
and the creation of a dedicated role or team may be necessary.

Unfortunately, lots of the fluids that surgical instruments come into contact with on a daily basis are also their key enemies. Blood, pus, surgical debris, dish or laundry soap, bleach, iodine-type and chlorhexidine solutions are all primary causes of staining and pitting of instruments.

The washing process should begin within 20 minutes after surgery, even if sterilization will take place much later. Only solutions that specifically state 'for surgical instruments' on the label should be used. After cleaning all items should be visually inspected, including all surfaces, cannulations, joints, lumens and ratchets.

\section{STERILIZATION OF INSTRUMENTS}

All surgical instruments need to be sterilized ready for surgical procedures. They must be clean and free from blood before being placed in the autoclave, as the temperature $\left(121-132^{\circ} \mathrm{C}\right)$ will cause chemical reactions that can make the stain permanent.

Instruments should be sterilized with their ratchets open to allow better steam penetration and prevent the box joints from cracking. If packed within a tray, then it should be perforated to allow better steam penetration and more effective drying. For efficiency, heavy instruments should be placed at the bottom and lighter, more delicate instruments on top. If sterilizing in paper or plastic pouches, they should not be stacked during sterilization and, if possible, pouches should be sterilized on their side. It is important that the stated maximum load for the autoclave is not exceeded.

\section{MAINTENANCE}

A maintenance plan should include servicing, restoration and sharpening of instruments on a regular basis and in a proactive approach. All members of the team should have the knowledge to be able to identify faults to remove those items from general use.

\section{KEY LEARNING OBJECTIVES}

Analyse current methods of cleaning to ensure the correct methods are being used

- Understand how regular care and maintenance prolong the lifespan of instruments

- Identify common faults in instruments and equipment and how to resolve them

\section{MULTIPLE CHOICE QUESTIONS}

1. What product should be used to clean surgical instruments after a procedure?
(A) Chlorhexidine solutions
(B) Washing-up liquid
(C) Soap (pH7-8)
(D) Cold soak solution

2. How should instruments with box joints be sterilized?

(A) With box joint open

(B) With box joint fully closed

(C) With box joint closed on first click

(D) Does not matter

3. When should instruments be checked for damage?
(A) By the surgeon at the beginning of the procedure
(B) Prior to washing the instrument
(C) After washing but prior to sterilization
(D) After sterilization

\section{Surgery into the beyond: how to mend a broken heart}

\section{Alison Young}

It is estimated that approximately $10 \%$ of dogs presented to primary care veterinary practices have heart disease, and chronic valvular heart disease (CHVD) is the most common heart disease of dogs in many parts of the world, accounting for approximately $75 \%$ of canine cases of heart disease cases seen by veterinary practices in North America.

\section{MITRAL VALVE DISEASE}

Myxomatous mitral valve disease is usually a degenerative condition. The valve edges become thickened and allow leakage of blood back into the atrium. The chordae tendineae, sometimes referred to as the 'heart strings' since they resemble small pieces of string, attach to the papillary muscles and hold the atrioventricular valves in place while the heart is pumping. The chordae tendineae can become elongated and even rupture, causing the valve edges to be pulled even further away from each other and allowing even more blood to regurgitate back into the atrium.

\section{MEDICAL MANAGEMENT}

Many dogs with early disease do not, and may never, require treatment for their disease. Dogs that develop signs of failure are very likely to ultimately succumb to their disease.

\section{SURGICAL TREATMENT - MITRAL VALVE REPAIR}

The aim of surgery is to reduce the volume of leakage through the valve, which will also enable the heart to 